\title{
Optical Properties of Negative Index Materials
}

\author{
J. P. Pandey \\ Department of Physics, M. L. K. P. G. College, Balrampur (UP), India
}

\begin{abstract}
In this communication, the optical properties i.e. the reflection behavior of one-dimensional PCs containing left-handed materials (LHMs) are theoretically investigated. The left-handed materials (LHMs) also called negative index materials (NIMs) or double negative (DNG) materials are artificial composite with both negative permittivity $\varepsilon$ and permeability $\mu$. The effect of variation of refractive index and number of periodic

layers on the reflection behavior of PIM-PIM and PIM-NIM structure is studied here. The reflection behavior is compared to the normal photonic crystal structure that consists of alternate layers of dielectric materials having positive index of refraction (PIM-PIM) and structure containing periodic arrangement of PIM and NIM. From the study, it is observed that the reflection band gap increases in PIM-NIM structure as compared to PIMPIM structure.
\end{abstract}

\section{Introduction}

A photonic crystal is a material in which the refractive index is modulated on a length scale compared to the desired operation wavelength of electromagnetic spectrum. It is said to be a crystal because it is formed by periodic arrangement of basic building blocks of refractive indices. The term photonic is added since photonic crystals are designed to affect the propagation propertied of photons. In general, the wave propagation is importantly affected when it enters into a material where some features that concern this wave are modulated. The behavior of photon with a certain frequency will depend on the propagation direction within the photonic crystal. The modulation of refractive index will cause that certain energies and direction is forbidden for photons. A region of frequency where a photonic crystal does not allow photons to propagate regardless of their direction and polarization is called a complete photonic band gap.

Photonic Crystals are the periodic layered structures of different type of dielectric materials with contrast refractive indices on the scale of wavelength radiation. Photonic crystals exhibit many unique features and have been gaining attention in the field of solid state and optical physics for making opto-electronic devices $[1,2]$. Shadrivov et al. [3] have purposed a complete band gap in one-dimensional left handed periodic structures. It is believed that modulation of refractive index in all three spatial directions is required to find a complete band gap and prevent the propagation of electromagnetic waves in all directions. So, they discovered and proposed the tri-layer of 1-D periodic structure containing left handed material that can trap light in three dimensional spaces due to the existence of a complete band gap. The transmission and omni-directional behavior of ternary layers of one-dimensional left handed photonic crystal have been studied by different researcher groups like K. Tang et al. [4], L. An et al. [5] and H.X. Da et al. [6]. M. Bloemer et al. [7] have proposed broad band omni-directional refractor from single layer of negative index material using experimental data [8]. They have shown that the operation bandwidth can be found $100 \%$ by increasing both electric and magnetic plasma frequencies of NIM. C. Nicolae et al. [9] have demonstrated that photonic superlattices consisting of periodic distribution of PIM and NIM superlattices have zero-averaged index band gap. Such band gap is invariant to the geometrical scaling of the superlattices or the direction of wave propagation in the superlattices. H. Jiang et al. [10] have shown the property of one dimensional photonic crystals with an $\varepsilon$ negative and a $\mu$-negative defect. With suitable parameters, the pair defect is equivalent to a transparent material with zero effective refractive index. Due to increasing the pair of defect, the gap-edge field can be a highly localized wave instead of the usual standing wave. G N Pandey et al. theoretically studied omni-directional reflection bands in one dimensional plasma photonic crystal in TE- and TM-modes. They also studied the reflectance and transmittance behaviors of superconductor dielectric photonic crystal at different incidence angles and temperature [11-14]. J P Pandey also studied about the enlargement of omnidirectional bandgaps in various PCs [15-16].

In this paper, the reflection properties of normal photonic crystal structure that consists of alternate layers of dielectric materials having positive index of refraction (PIM-PIM) and structure containing periodic arrangement of PIM and NIM are investigated. Further, the effect of variation of refractive index as well as the number of periodic layers on the reflection properties of PIM-NIM structure as compared to PIM-PIM is also observed. 


\section{Theoretical Background Using Transfer Matrix Method}

A one dimensional multilayer system is considered here which is composed of periodic arrays of two different materials with refractive index $n_{1}$ and $n_{2}$ and thickness of the layer $d_{1}$ and $d_{2}$ having propagation of EM wave along $\mathrm{x}$-axis normal to the interface. The refractive index of the structure is given by

$$
n(x)=\left\{\begin{array}{l}
n_{1}, 0<x<d_{1} \\
n_{2}, d_{1}<x<d
\end{array}\right.
$$

With $n(x)=n(x+d)$. Here, $d=d_{1}+d_{2}$ is the period of the lattice (or lattice constant). The electromagnetic field distribution within each layer can be expressed as the sum of right and left-hand side propagating wave.

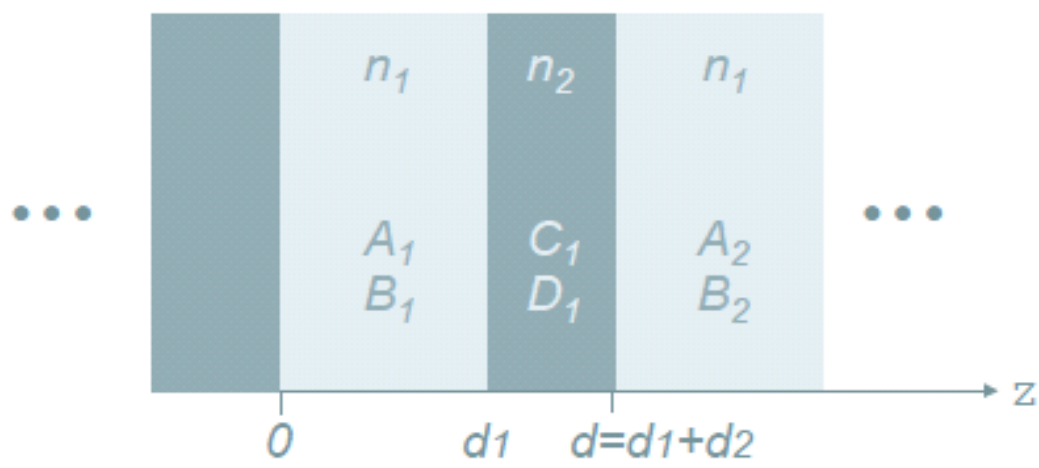

Figure 1: Schematic diagram of 1-D multilayer structure

In the both layers of the nth unit cell, the electric field can be written as $[17,18]$.

$$
\begin{aligned}
& E_{1}(x)=\left[\left[a_{n} e^{-i k_{1}(x-n d}\right)+b_{n} e^{i k_{1}(x-n d)}\right] e^{i \omega t} \\
& E_{2}(x)=\left[\left(c_{n} e^{-i k_{2}(x-n d}\right)+d_{n} e^{i k_{2}(x-n d)}\right] e^{i \omega t}
\end{aligned}
$$

where $k_{i}=\left[\left(\frac{n_{i} \omega}{c}\right)^{2}-\beta^{2}\right]^{1 / 2}=\frac{n_{i} \omega}{c} \cos \theta_{i} ; \theta_{i}$ is the ray angle in the $i^{\text {th }}$ layer $(i=1,2), \beta$ is the constant of propagation and $n_{i}=\sqrt{\varepsilon_{i} \mu_{i}}$, where $\varepsilon_{i}$ and $\mu_{i}$ are the dielectric permittivity and magnetic permeability of the constituent layers.

The coefficients $a_{n}, b_{n}, c_{n}$ and $d_{n}$ are related through the continuity conditions at the interfaces $x=(n-1) d$ and $x$ $=(n-1) d+d_{2}$. According to the continuity, it condition leads to the matrix equations, which relates the first layer coefficient of the nth cell, and is given as

$$
\left[\begin{array}{l}
a_{n-1} \\
b_{n-1}
\end{array}\right]=T_{n}\left[\begin{array}{l}
a_{n} \\
b_{n}
\end{array}\right]
$$

Where $\mathrm{T}_{\mathrm{n}}$ is called the transfer matrix and is given by

$$
T_{n}=\left[\begin{array}{ll}
A & B \\
C & D
\end{array}\right]
$$

The elements in the matrix A, B, C and D are

$$
\begin{gathered}
A=e^{i k_{1} d_{1}}\left[\cos k_{2} d_{2}+\frac{1}{2} i\left(\eta+\frac{1}{\eta}\right) \sin k_{2} d_{2}\right] ; \\
B=e^{-i k_{1} d_{1}}\left[\frac{1}{2} i\left(\eta-\frac{1}{\eta}\right) \sin k_{2} d_{2}\right]
\end{gathered}
$$




$$
\begin{aligned}
C=e^{i k_{1} d_{1}} & {\left[-\frac{1}{2} i\left(\eta-\frac{1}{\eta}\right) \sin k_{2} d_{2}\right] } \\
D & =e^{-i k_{1} d_{1}}\left[\cos k_{2} b-\frac{1}{2} i\left(\eta+\frac{1}{\eta}\right) \sin k_{2} d_{2}\right]
\end{aligned}
$$

The parameter $\eta$ depends on the polarization. Polarizations, $\eta$ for TE and TM is given by

$$
\eta_{T E}=\frac{k_{1}}{k_{2}} \quad \text { and } \quad \eta_{T M}=\frac{k_{1} n_{2}{ }^{2}}{k_{2} n_{1}{ }^{2}}
$$

The coefficient of right and left hand side propagating wave in both sides of the multiplayer structure $a_{N}$ and $b_{N}$ for the finite stacks, are calculated by multiplying transfer matrix of each cell as

$$
\left[\begin{array}{l}
a_{0} \\
b_{0}
\end{array}\right]=T_{1} T_{2} \ldots . . T_{N}\left[\begin{array}{l}
a_{N} \\
b_{N}
\end{array}\right] \text {, }
$$

Where $\mathrm{N}=$ total numbe $\mathrm{r}$ of the cell. By solving above matrix equation with the condition $\mathrm{b}_{\mathrm{N}}=0$, the coefficient of reflection is given as $r_{N}=\left(\frac{b_{0}}{a_{0}}\right)$.

Thus the reflectivity (or reflectance) of the structure is $\quad R_{N}=\left|r_{N}\right|^{2}$.

Now, according to Bloch theorem, the electric field vector is of the form $E=E_{K}(x) e^{i(\omega t-K x)}$, where $E_{K}(x)$ is periodic with the period ' $d$ '. For the determination of $K$ as a function of eigen value, we can write the equation as

$$
\left[\begin{array}{ll}
A & B \\
C & D
\end{array}\right]\left[\begin{array}{l}
a_{n} \\
b_{n}
\end{array}\right]=\quad e^{i K d}\left[\begin{array}{l}
a_{n} \\
b_{n}
\end{array}\right]
$$

The dispersion relation for the PC structure containing the alternate stacks of positive index materials (PIM) is given by

$$
K(\omega)=\left(\frac{1}{d}\right) \cos ^{-1}\left[\cos \left(k_{1} d_{1}\right) \cos \left(k_{2} d_{2}\right)-\frac{1}{2}\left(\eta+\frac{1}{\eta}\right) \sin \left(k_{1} d_{1}\right) \sin \left(k_{2} d_{2}\right)\right]
$$

The dispersion relation for the PC structure containing the alternate layer of positive and negative index material is given by

$$
K(\omega)=\left(\frac{1}{d}\right) \cos ^{-1}\left[\cos \left(k_{1} d_{1}\right) \cos \left(k_{2} d_{2}\right)+\frac{1}{2}\left(\eta+\frac{1}{\eta}\right) \sin \left(k_{1} d_{1}\right) \sin \left(k_{2} d_{2}\right)\right]
$$

\section{Results And Discussions:}

In this present communication, in order to study the reflection behavior in Photonic Crystals (PC), having alternate regions of PIM - PIM (Positive Index Materials - Positive Index Materials) and PIM - NIM (Positive Index Materials- Negative Index Materials) structure, the reflectivity of the proposed structure at different refractive indices and at various numbers of layers at zero angle of incidence for both polarizations (TE - \& TM) are calculated A graph is plotted between reflectivity and normalized frequency at different refractive indices and at different numbers of layers for the proposed structure which is shown graphically in Figs 2, 3, 4 and 5 .

In Fig 2(a), $\mathrm{n}_{1}=1.00$ and $\mathrm{n}_{2}=2.5$ is taken at zero angle of incidence for PIM-PIM structure and in Fig 2(b), $n_{1}=1.00$ and $n_{2}=3.5$ is taken for the same. From the figure, it is seen that the reflection band increases due to increase of refractive index. $\mathrm{n}_{1}=1.00$ and $\mathrm{n}_{2}=2.5 \& 3.5$ is also taken at zero angle of incidence for PIM-NIM structure in Figs 3(a-b). It is found that reflection band increases due to increase in refractive index. From the figures 2 and 3, it is also clear that the reflection band is much wider in PIN-NIM structure as compared to PIMPIM structure. It reveals from the above discussion that PIM-NIM structure can be used as a broad band reflector. In fig. $4, \mathrm{n}_{1}=1.36$ and $\mathrm{n}_{2}=3.5$ is taken at zero angle of incidence with variation in number of layers $\mathrm{N}=7$, 
$\mathrm{N}=12$ and $\mathrm{N}=18$ for PIM-PIM structure. From the figure, it is clear that the reflection band increases as the number of layers increase. In fig. $5, \mathrm{n}_{1}=1.36$ and $\mathrm{n}_{2}=3.5$ is considered at zero angle of incidence with variation in number of layers $\mathrm{N}=7, \mathrm{~N}=12$ and $\mathrm{N}=18$ for PIM-NIM structure. The reflection band increases as the number of layers increase. So, it is demonstrated from the figures 4 and 5 that the reflection band is much wider in PINNIM structure as compared to PIM-PIM structure with the variation of number of layers. From the above discussion, it is concluded that PIM-NIM structure can be used as a broad band reflector.
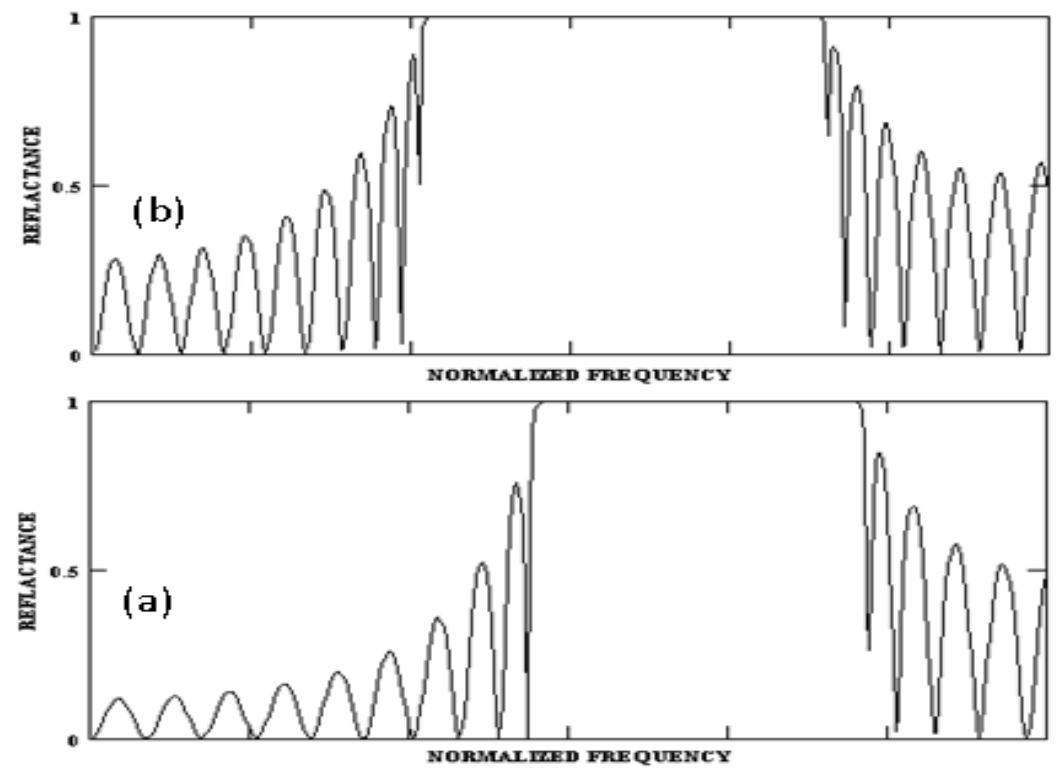

Fig: 2(a) PIM-PIM with angle fixed at $0^{0}$ and $\mathrm{n}_{1}=1.00, \mathrm{n}_{2}=2.5$ and fig.2 (b) with $\mathrm{n}_{1}=1.00, \mathrm{n}_{2}=3.5$
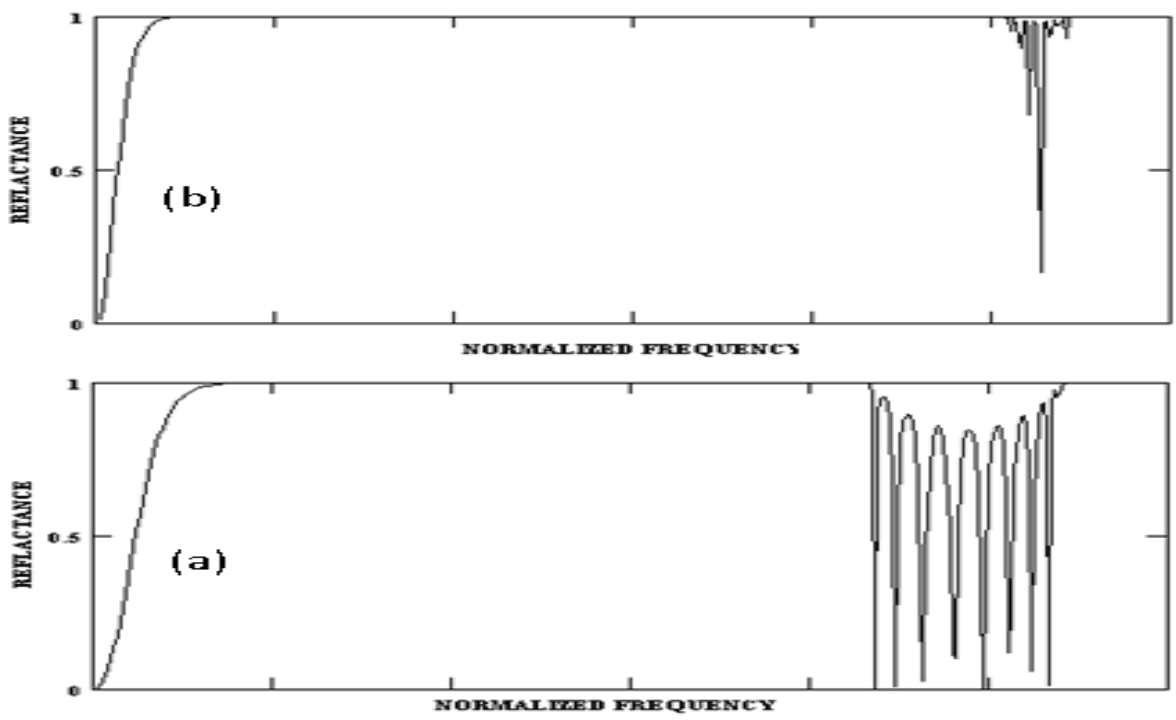

Fig.3 (a) PIM-NIM with angle fixed at $0^{0}$ and $\mathrm{n}_{1}=1.00, \mathrm{n}_{2}=2.5$ and Fig.3 (b) $\mathrm{n}_{1}=1.00, \mathrm{n}_{2}=3.5$ 

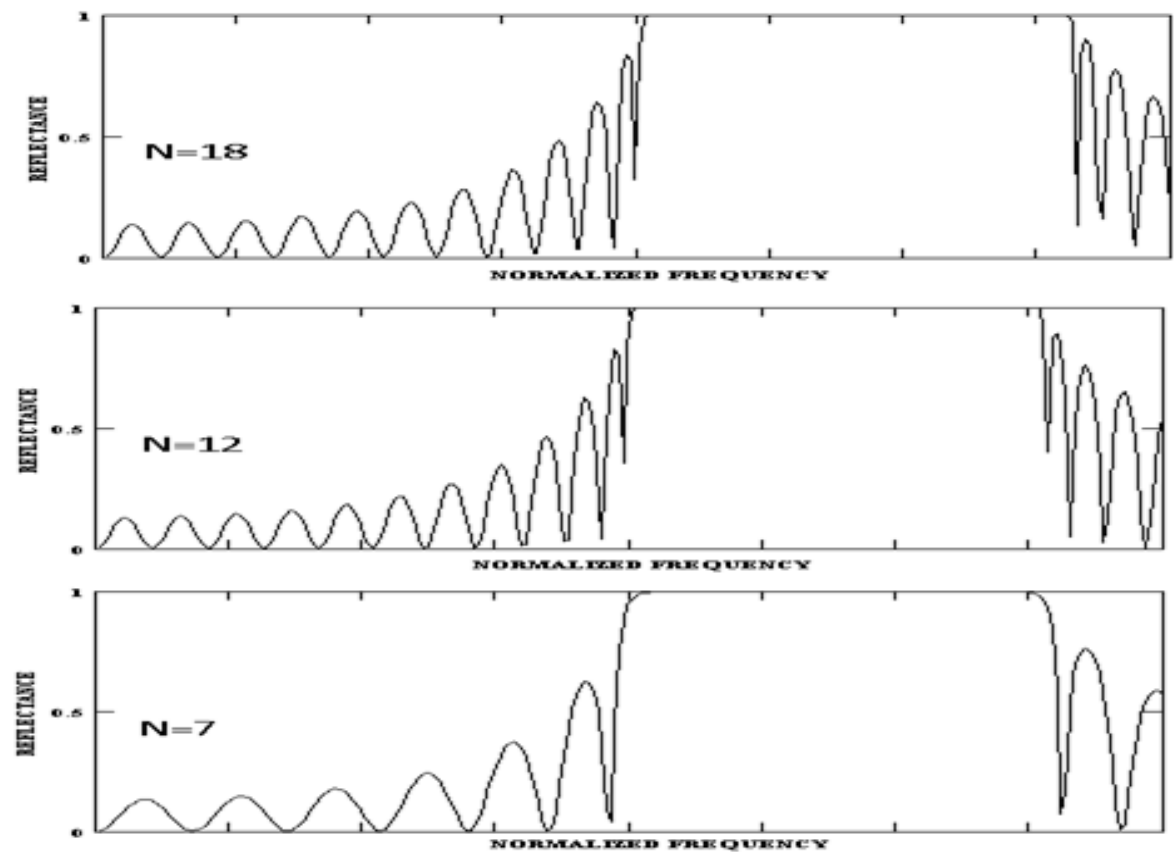

Fig. 4 PIM-PIM structure having fixed angle at $0^{0}$ and $\mathrm{n}_{1}=1.36, \mathrm{n}_{2}=3.5$ with variation in number of layers $\mathrm{N}=7$, $\mathrm{N}=12$ \& $\mathrm{N}=18$.
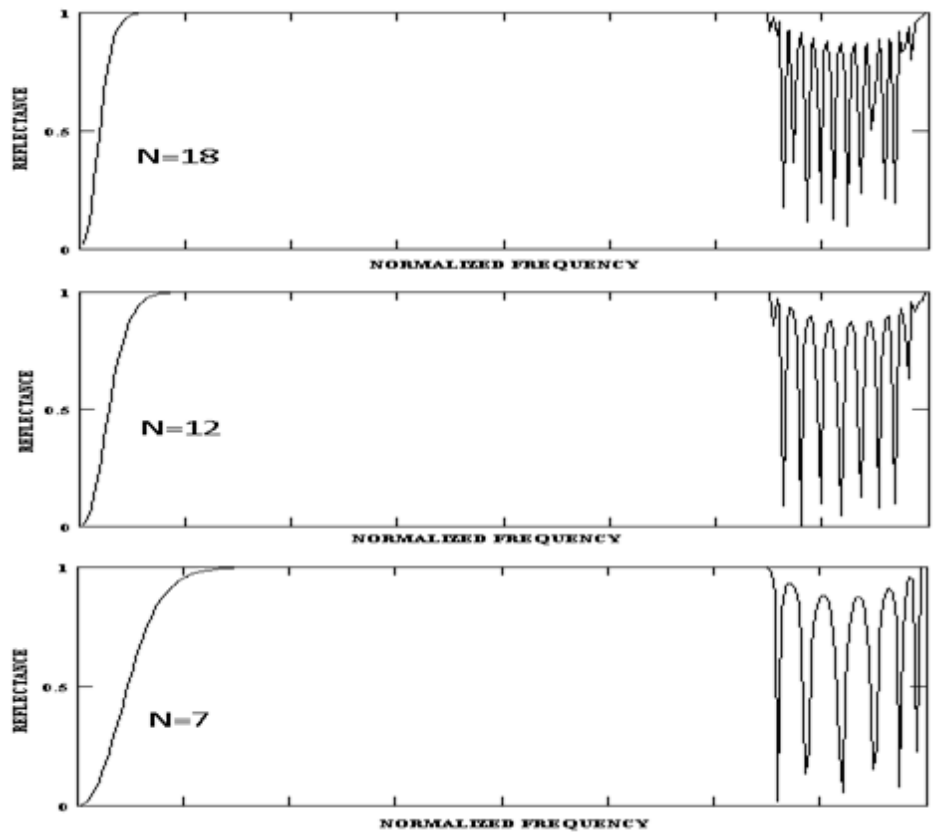

Fig.5. PIM-NIM structure having fixed angle at $0^{0}$ and $\mathrm{n}_{1}=1.36, \mathrm{n}_{2}=3.5$ with variation in number of layers $\mathrm{N}=7$, $\mathrm{N}=12$ \& $\mathrm{N}=18$.

\section{References:}

[1]. J. D. Joannopoulos, R. D. Meade and J. N. Winn, “Photonic Crystals”, Princeton University Press, Princeton (1995).

[2]. M. Scalora, J. P. Dowling, C. M. Bowden, and M. J. Bloemer, Phys. Rev. Lett., 73, 1368 (1994).

[3]. Ilya V. Shadrivov, Andrey A. Sukhorukov, and Yuri S. Kivshar, Phys. Rev. Lett. 95, 193903, (2005).

[4]. K. Tang, Y. Xiang, S. Wen, Proc. SPIE, 6020, 183 (2005).

[5]. L. An and G.P. Wang, Chin. Phys. Lett., 23, 388 (2005).

[6]. H.X. Da, C. Xu and Z.Y. Li, Phys. Lett. A., 345, 459 (2005).

[7]. M. Bloemer, G.D’Aguanno, M. Scalora and N. Mattiucci, 87, 261921 (2005).

[8]. R.A. Shelby, D. R. Smith and S. Schultz, 77 (2001).

[9]. Nicolae C. Panoiu and Richard M. Osgood, Jr., S Zhang and S. R. J. Brueck, J. Opt. Soc. Am. B, 23, 506 (2006).

[10]. H. Jiang, H. Chen and S. Zhu, Phys. Rev. E., 79, 0466601 (2006).

[11]. G.N. Pandey, K. B. Thapa and S.P. Ojha, Optik - International Journal for Light and Electron Optics, Volume 124, Issue 18, 33963401 (Sept. 2013). 
[12]. G. N. Pandey and S. P. Ojha, Optik - International Journal for Light and Electron Optics, Volume 124, Issue 18, 3514-3519 (Sept. 2013).

[13]. G. N. Pandey, khem B Thapa and S. P. Ojha, Optik - International Journal for Light and Electron Optics, Volume 125, 252-256 (2014).

[14]. G. N. Pandey, International Journal of Advances in Electronic and Electronic Engineering, Vol. 3, No.8, Page No 965-970, (2013).

[15]. J P Pandey, International Journal of Pure and Applied Physics (IJPAP), Vol 13, Number 2 pp. 167-173 (2017).

[16]. J P Pandey, IOSR Journal of Applied Physics (IOSR-JAP), Volume 9, Issue 2, pp 59-63 Ver. I (Mar. - Apr. 2017).

[17]. M. Born and E. Wolf, Principles of Optics, New York: Pergamon (1980).

[18]. P. Yeh, Optical Waves in Layered Media, New York: John Wiley and Sons (1988). 